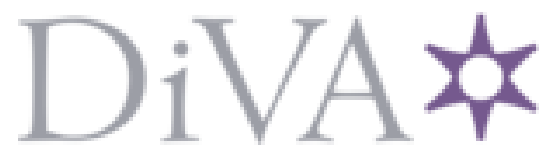

http://www.diva-portal.org

This is the published version of a paper published in Physical Review A. Atomic, Molecular, and Optical Physics.

Citation for the original published paper (version of record):

Kolliopoulos, G., Bergues, B., Schröder, H., Carpeggiani, P A., Veisz, L. et al. (2014)

Revealing quantum path details in high-field physics.

Physical Review A. Atomic, Molecular, and Optical Physics, 90(1): 013822

https://doi.org/10.1103/PhysRevA.90.013822

Access to the published version may require subscription.

N.B. When citing this work, cite the original published paper.

Permanent link to this version:

http://urn.kb.se/resolve?urn=urn:nbn:se:umu:diva- 134420 


\title{
Revealing quantum path details in high-field physics
}

\author{
G. Kolliopoulos, ${ }^{1,2}$ B. Bergues, ${ }^{3}$ H. Schröder,${ }^{3}$ P. A. Carpeggiani, ${ }^{1,2}$ L. Veisz, ${ }^{3}$ G. D. Tsakiris, ${ }^{3}$ \\ D. Charalambidis, ${ }^{1,2}$ and P. Tzallas ${ }^{1, *}$ \\ ${ }^{1}$ Foundation for Research and Technology-Hellas, Institute of Electronic Structure \& Laser, P.O. Box 1527, \\ GR-71110 Heraklion (Crete), Greece \\ ${ }^{2}$ Department of Physics, University of Crete, P.O. Box 2208, GR-71003 Heraklion (Crete), Greece \\ ${ }^{3}$ Max-Planck-Institut für Quantenoptik, D-85748 Garching, Germany
}

(Received 19 October 2013; published 18 July 2014)

\begin{abstract}
The fundamental mechanism underlying harmonic emission in the strong-field regime is governed by tunnel ionization of the atom, followed by the motion of the electron wave packet in the continuum, and finally by its recollision with the atomic core. Due to the quantum nature of the process, the properties of the electron wave packet strongly correlate with those of the emitted radiation. Here, by spatially resolving the interference pattern generated by overlapping the harmonic radiation emitted by different interfering electron quantum paths, we have succeeded in unravelling the intricacies associated with the recollision process. This has been achieved by mapping the spatial extreme-ultraviolet (EUV)-intensity distribution onto a spatial ion distribution, produced in the EUV focal area through the linear and nonlinear processes of atoms. By in situ manipulation of the intensity-dependent motion of the electron wave packets, we have been able to directly measure the difference between the harmonic emission times and electron path lengths resulting from different electron trajectories. Due to the high degree of accuracy that the present approach provides, we have been able to demonstrate the quantum nature of the recollision process. This is done by quantitatively correlating the photoemission time and the electron quantum path-length differences, taking into account the energy-momentum transfer from the driving laser field into the system. This information paves the way for electron-photon correlation studies at the attosecond time scale, while it puts the recollision process from the semiclassical prospective into a full quantum-mechanical context.
\end{abstract}

DOI: 10.1103/PhysRevA.90.013822

PACS number(s): 42.65.Ky, 42.65.Re, 32.80.Rm

\section{INTRODUCTION}

Strong-field light-matter interactions induced by intense laser sources led to the observation of a broad range of phenomena such as the generation of coherent extremeultraviolet (EUV) radiation and attosecond pulse formation (see Refs. [1-5] and references therein). High-order harmonics have been used for imaging atomic [6] and molecular orbitals [7-9] while the use of attosecond pulses have pushed the temporal resolution of ultrafast dynamical studies towards the scale of the atomic unit of time. New pulse characterization techniques have been developed for measuring the duration of these pulses [1,3], which have been successfully utilized in the observation of a number of new processes in all states of matter $[3,4,10-12]$. At the same time, frequency combs have been recently developed in the EUV spectral region [13-15], pushing the frequency resolution in the $\mathrm{MHz}$ range, thus improving the precision of the measurements by an order of magnitude $[14,15]$. Thus, the detailed study of the strong-field light-matter interaction is essential for an in-depth understanding of the harmonic generation mechanism and the further development of the above-mentioned research directions. The aim of the present work is to experimentally reveal the intricacies which are associated with the high-orderharmonic generation process occurring during the interaction of noble gases with intense infrared (IR) laser pulses. The tunneling of an electron through a suppressed atomic potential,

*Corresponding author: ptzallas@ iesl.forth.gr followed by its motion in the continuum, is the fundamental mechanism underlying strong-field laser-atom interactions [16-21]. In the spirit of the "three-step model" [18,20,21], the IR field suppresses the atomic potential and allows the valence electron to tunnel through. The electron moves almost freely in the driving field, gaining kinetic energy, which is converted to photons upon its recombination. Due to its quantum nature, the interaction is influenced by the phase of the released electron wave packets. This has been experimentally demonstrated using spectroscopic [22-24] and interferometric approaches [25]. Thus, detailed mapping of the electron wave-packet interference patterns provides an essential insight into the physics underlying the interaction. A process providing access to the complexities of the interaction is the generation of highorder harmonics of the laser frequency. The phase-amplitude distribution of the emitted EUV carries all the information about the harmonic generation process and vice versa. Thus, the visualization of the EUV-spatial-amplitude distribution, as it results from interfering electron wave-packet contributions, is of crucial importance. The spatially integrating measurement approaches applied so far have impeded this accomplishment, as they average out the phase effects in the generation process [22-24].

In this article, we demonstrate a method which overcomes this obstacle. An EUV-spatial-amplitude-distribution image is deduced from the imprint on the measured spatial distribution of ions, produced through EUV-photon ionization of atoms. This image exhibits an interference pattern due to the overlapping of the harmonic radiation emitted by different interfering electron quantum paths. The interference 
patterns in the image carry phase information about the interfering electron wave packets. By mapping the dependence of the interference pattern onto the intensity of the driving field, we have managed to measure the difference between the harmonic emission times and the electron path lengths associated with different electron trajectories. The dependence of the interference pattern and the harmonic yield on the laser intensity demonstrates that the classical treatment of the interaction does not suffice to describe all the details of the energy transfer from the field to the electron, as quantized energy transfer is imprinted in the present measurements. This information has been retrieved by quantitatively correlating the photoemission time and electron quantum path-length differences due to the energy transfer of the IR laser field into the system.

\section{MAPPING THE ELECTRON WAVE-PACKET INTERFERENCES}

The high-order-harmonic generation process at the singleatom level is governed by the electron quantum path interference (see Refs. [18-24] and references therein) and on the macroscopic scale, by phase-matching conditions [26-30], i.e., $\vec{k}_{q}=q \vec{k}+\Delta \vec{k}_{g}+\Delta \vec{k}_{d}+\vec{\nabla} \phi_{q}^{L, S}\left(I_{\ell}\right)$, with $\vec{k}_{q}$ and $\vec{k}$ being the $k$ vectors of the $q$ th harmonic and the fundamental, respectively, $\Delta \vec{k}_{g}$ the Gouy phase shift, $\Delta \vec{k}_{d}$ the mismatch caused by dispersion, and $\phi_{q}^{L, S}\left(I_{\ell}\right)$ is the phase accumulated by the electron wave packets during their motion in the continuum. The phase-amplitude distribution of the emitted harmonics strongly depends on the harmonic order $(q)$, the intensity of the driving field $\left(I_{\ell}\right)$, the focusing conditions, and the dispersion properties of the medium. Thus, at fixed focusing conditions, the phase-amplitude distribution of the emitted harmonics is influenced mainly by the intensity-dependent phase $\phi_{q}^{L, S}\left(I_{\ell}\right)$. Regarding the dependence of the EUV-phaseamplitude distribution on $q$ for the plateau harmonics, two intensity-dependent quantum interfering electron trajectories, the long $(L)$ and the short $(S)$, with different flight times, contribute. This is due to gradient forces induced by the driving field onto the electron trajectories, primarily to the off-axis and on-axis harmonic emission [29,31-33] with phases $\phi_{q}^{L}\left(I_{\ell}\right)$ and $\phi_{q}^{S}\left(I_{\ell}\right)$, respectively. In the deep cutoff spectral region the two trajectories degenerate into one with a single phase.

The access to the harmonic generation mechanism has been achieved by spatially resolving the interference pattern created by overlapping at focus the on- and off-axis harmonics generated by the nonlinear interaction of a Ti:sapphire fs laser pulse with xenon gas. In this case, an EUV-spatial-amplitudedistribution image is deduced from its imprint on the measured spatial distribution of ions produced through EUV-photon ionization of atoms. The experiment was performed utilizing a $10 \mathrm{~Hz}$ repetition rate Ti:sapphire laser system delivering pulses of up to $170 \mathrm{~mJ}$ energy, $\tau_{L}=33$ fs duration, and a wavelength of $800 \mathrm{~nm}$ (IR). The experimental setup is shown in Fig. 1(a). An annular laser beam (formed using a super-Gaussian beam stop) with an outer diameter of $\approx 2.5 \mathrm{~cm}$ and energy of $\approx 15 \mathrm{~mJ} /$ pulse was focused with a $f=3 \mathrm{~m}$ lens into a pulsed gas jet (P-GJ) filled with Xe, where the harmonic radiation was generated. In this focusing geometry the confocal parameter of the laser beam $(\approx 10 \mathrm{~cm})$ is approximately two orders of magnitude longer compared to the length of the Xe gas medium $(\approx 1 \mathrm{~mm})$. Thus, the intensity of the driving field along the propagation in the Xe gas can be considered as constant. After the jet, a Si plate was placed at Brewster's angle for the fundamental $\left(75^{\circ}\right)$ to reflect the harmonics [34] towards the detection area while substantially attenuating the IR field. The EUV radiation, after reflection from the $\mathrm{Si}$ plate, passes through a 5-mm-diam aperture (A) which blocks the residual outer part of the IR beam. Subsequently, the EUV beam was focused into the target gas jet (T-GJ) by a spherical gold mirror (SM) of $5 \mathrm{~cm}$ focal length. Argon and helium gases were introduced in the EUV-atom interaction volume where single- and two-photon ionization occurs by the 11 th to 15 th harmonics. In the case of using argon in the detection jet, the contribution of the two-EUV-ionization processes has a negligible influence on the $\mathrm{Ar}^{+}$signal. This has been clarified by the absence of the $\mathrm{Ar}^{2+}$ signal (induced by a two-photon direct-double ionization scheme) in the recorded ion-mass spectrum. Also, any two-color (IR-EUV) two-photon ionization process can be further excluded due to the elimination of the IR radiation in the detection region. This is verified by the absence of "side bands" in the energy-resolved single EUV photon ionization photoelectron spectrum of Ar. When helium is used as a target gas, the harmonic generation conditions have been maximized, reaching an energy of $\approx 100 \mathrm{~nJ}$ on the target, and the $\mathrm{He}^{+}$detection and collection efficiency conditions have been optimized. These optimizations were not necessary in the case of using $\mathrm{Ar}$ in the target jet since the $\mathrm{Ar}^{+}$signal was orders of magnitude stronger compared to $\mathrm{He}^{+}$and thus was recordable under much less demanding conditions due to the linearity of the ionization scheme. Care has been taken to fix the angle of incidence of the EUV beam on the gold mirror at $0^{\circ}$. The EUV images were monitored by means of a high spatial resolution $(1 \mu \mathrm{m})$ ion-imaging detector (I-ID) $[35,36]$, which records the spatial distribution of the ionization products resulting from the interaction of the EUV light with the target gas. The spectrum of the EUV radiation in the interaction area was determined by measuring the energyresolved, single-photon ionization, photoelectron spectra of Ar gas. The electron spectra were recorded using a $\mu$-metal shielded time-of-flight (TOF) ion or electron spectrometer, attached to a second EUV beam-line arm [note that the TOF arm is not shown in Fig. 1(a)]. In order to have the same experimental conditions in both the TOF and the I-ID setups, the TOF arm was constructed in an identical way to the one of the I-ID. This is done by using a second Si plate (mounted on a translation stage) placed at Brewster's angle for the fundamental $\left(75^{\circ}\right)$. The length of the second arm, the aperture, and the spherical gold mirror were the same as those used in the I-ID arm. For the measurement of the photoelectron spectrum in the interaction region the focused EUV beam was tilted by $\approx 1.5^{\circ}$ with respect to the incoming beam. This is done in order to avoid including the photoelectrons that resulted from the incoming EUV beam. In this case, while all the generated harmonics are entering into the interaction area, only the harmonics above the 11th can ionize the Ar gas by single-photon absorption. The signal of the 11 th, 13 th, and 15 th harmonics after the reflection from the spherical gold mirror 


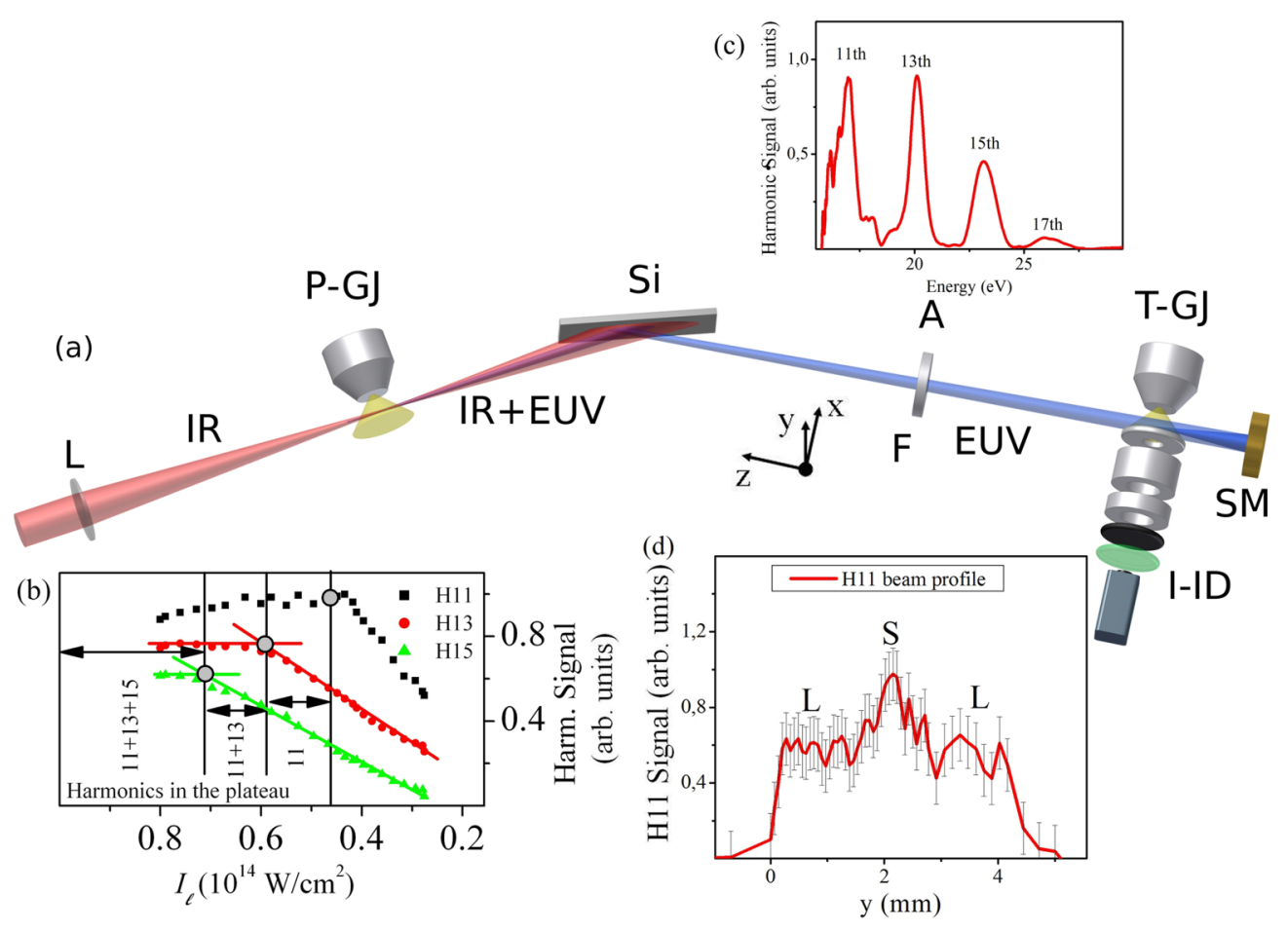

FIG. 1. (Color online) (a) Experimental setup used for imaging the EUV focus interference pattern. L: IR focusing lens. P-GJ: Harmonic production gas jet filled with xenon. A: Aperture. F: Thin metal filter. SM: Gold spherical mirror. T-GJ: Target gas jet filled with argon. I-ID: Ion imaging detector. (b) The dependence of the harmonic signal on $I_{\ell}$. The harmonic signal has been recorded up to $I_{\ell}$ values at which all the harmonics are in the plateau region. The vertical black lines depict the harmonic cutoff regions. (c) Typical harmonic spectrum recorded at $I_{\ell}$ $\approx 7 \times 10^{13} \mathrm{~W} / \mathrm{cm}^{2}$. (d) Beam profile of the 11 th harmonic on the surface of the gold spherical mirror determined by means of the knife-edge technique. The beam profile and the diameter of the 13th and 15th harmonics [for simplicity, not shown in (d)] are approximately the same with the 11 th.

is shown in arbitrary units in Fig. 1(b). Figure 1(c) shows a typical harmonic spectrum recorded at $I_{\ell} \approx 7 \times 10^{13} \mathrm{~W} / \mathrm{cm}^{2}$. The dependence of the harmonic signal on $I_{\ell}$ has been used for the measurement of the harmonic amplitudes $\left(E_{q}\right)$ and the determination of the cutoff regions. The 17 th harmonic has been detected (at high $I_{\ell}$ ) with an order-of-magnitude smaller amplitude than the 11th harmonic. All harmonics higher than the 17th have even smaller amplitudes and thus were not detectable. The energy of the EUV radiation has been measured by means of an XUV calibrated photodiode, which has been placed after the aperture. At the maximum laser intensities $\left(I_{\ell_{\max }} \approx 10^{14} \mathrm{~W} / \mathrm{cm}^{2}\right)$ used, the outer $4.6-\mathrm{mm}$ EUV beam diameter on the focusing gold mirror and the $2 \pm$ $1 \mu \mathrm{m}$ EUV focal spot diameter have been measured using the I-ID. The beam diameters on the surface of the gold mirror for the harmonics emitted by the "short" and "long" trajectories were also determined by means of the knife-edge technique [Fig. 1(d)]. The spatial resolution of the I-ID and the shot-toshot point stability of the EUV at the focus contributes to the $\pm 1 \mu \mathrm{m}$ error in the measured focal spot diameter.

In order to show how a spatially resolved EUV-interference pattern appears when the on- and off-axis emitted harmonics overlap at the focus, we have calculated the image of the focused EUV beam (harmonics from 11th up to 15th) for two different values of $I_{\ell}$. Figure 2(a) shows the profiles of the plateau harmonics on the surface of the focusing mirror. The outer part of the beam contains mainly radiation resulting from the "long" electron trajectories while the inner part by the "short" [31]. Figures 2(b) and 2(c) show the images of the focused harmonics generated at two $I_{\ell}$ values, for which harmonic phase distributions lead to on-axis $(z)$ destructive [Fig. 2(b)] and constructive [Fig. 2(c)] interference at the focus position with a visible double- and single-peak structure, respectively. The single- and double-peak structures occur when the phase difference between the trajectories is $\Delta \varphi_{q}^{S, L} \approx$ $2 n \pi$ and $\Delta \varphi_{q}^{S, L} \approx(2 n+1) \pi$, respectively $(n=0,1,2, \ldots)$. The calculated EUV focus images are obtained by the Debye integral [37], after applying the Huygens-Fresnel principle on a spherical mirror with a $10 \mathrm{~cm}$ radius of curvature. The spectral phase distribution and the relative amplitudes between the "short" and "long" trajectory harmonics have been calculated from the single-atom, three-step, quantum-mechanical model [18]. The relative amplitudes between different harmonics have been taken from the graph of Fig. 1(b) which shows the dependence of the harmonic yield on $I_{\ell}$. In the calculations, for laser intensities giving rise to harmonic emission in the plateau, the beam diameters on surface of the mirror for the harmonics emitted by the "short" and "long" trajectories were taken from Fig. 1(d). The diameter was found to be approximately the same for all harmonics in the plateau. The experimental observation of this interference pattern is the key point of the present study since it provides the spatial EUV-amplitude distribution and spatial positions at which the "long"-"short" trajectory phase differences become $2 n \pi$ 
(a)

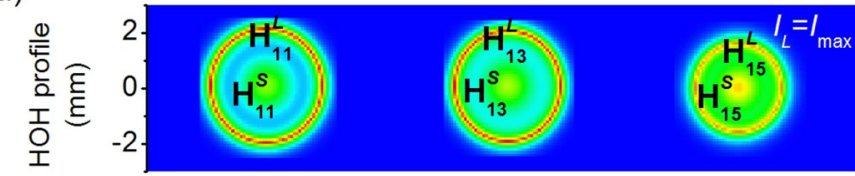

(b)

(c)

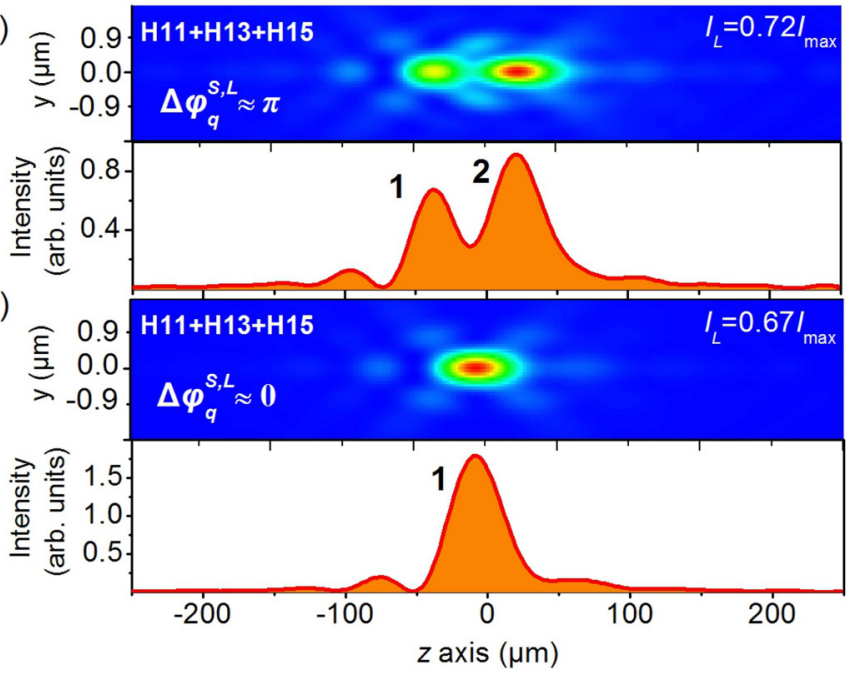

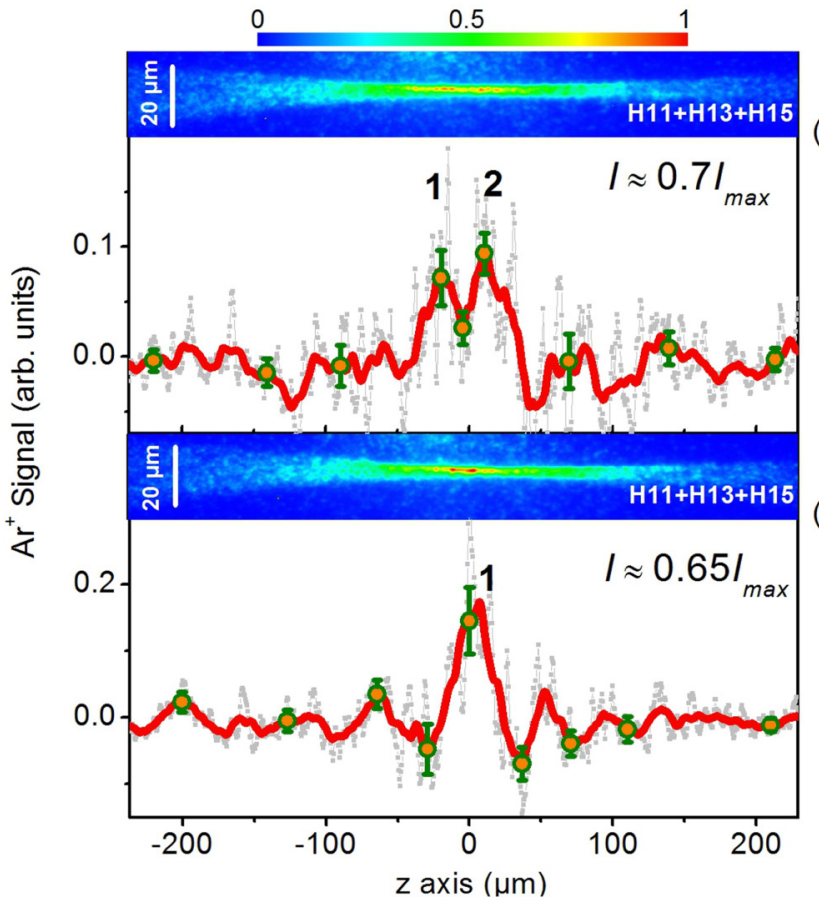

(d)

(e)

FIG. 2. (Color online) Spatial imaging the interference pattern created at the focus of the EUV beam. (a) Calculated profiles of the 11th, 13th, and 15th harmonics on the surface of the EUV focusing mirror, at $I_{\ell}=I_{\max }=10^{14} \mathrm{~W} / \mathrm{cm}^{2}$. (b), (c) Calculated images of the focused EUV beam for two different values of the drive laser intensity $I_{\ell}$, for both of which the harmonics are lying on the plateau spectral region having approximately equal relative amplitudes [see Fig. 1(b)]. At these intensities the phase distribution of the EUV beam leads to on-axis (z) (b) destructive and (c) constructive interference at the focus position with a double- and single-peak structure, respectively. (d), (e) EUV focus images produced by the single-photon ionization signal of argon at two different drive-laser intensities $I_{\ell}$. The relative amplitudes of the harmonics in the interaction region were approximately equal for both images [see Fig. 1(b)]. The red lines are the 30-point running average of the raw data and the error bars represent one standard deviation of the mean. 600 shots were accumulated for each image.

or $(2 n+1) \pi$ in the interaction area. Figures 2(d) and 2(e) show the ion distributions recorded at the EUV focus, at two different values of $I_{\ell}$, using argon as a target gas. To make the trend clearer, the interference pattern in Figs. 2(d) and 2(e) was retrieved after subtraction of the smooth part of the signal (background signal). The reasons why there is a limitation of the modulation depth of the interference pattern that does not affect the positions of the minima and maxima in the images are (i) the existence of a background signal which comes from the contribution of the out-of-plane signal in the projected focus image, (ii) the ion signal which is produced by the incoming (unfocused) EUV beam, and (iii) the finite resolution of the I-ID. The fringe visibility of the interference pattern is close to its optimum value after subtracting the background signal. Similar images have also been recorded using spectrally filtered EUV radiation. In these measurements, the EUV radiation was passing through a 150-nm-thick Sn filter [denoted with an "F" in Fig. 1(a)], which transmits only the 11th, 13th, and 15th harmonics. The characteristic interference patterns, exhibiting a double[Fig. 2(d)] and single-peak [Fig. 2(e)] structure around the focus, are in qualitative agreement with the characteristic features of Figs. 2(b) and 2(c), respectively.

As the electron quantum path interference is strongly dependent on $I_{\ell}$, we have performed a systematic measurement of the spatial ion distribution at the focus as a function of the driving intensity. This dependence is shown in the contour plot of Fig. 3(a). In this measurement, an intensity-dependent interference pattern along the $z$ axis was observed. This dependence is clearly shown as a modulation from a singlepeak structure to a double-peak structure in the normalized contour plot of Fig. 3(b), which clearly confirms the statistical relevance of the structures shown in Figs. 2(d) and 2(e). The "saw-type" structure is the result of the phase distribution of the harmonics due to the spatial dependence of the electron quantum path interference in the harmonic generation process. This structure was strongly pronounced at high $I_{\ell}$ values, where most of the harmonics lie in the plateau region. The structure is also observable at lower $I_{\ell}$ values where a transition of the harmonics from the plateau to the cutoff region takes place. A clear and intensity-independent, single-peak structure appears only at very low $I_{\ell}$ values, at which all the harmonics are evidently in the deep cutoff region. The measured structures are found to be in fair agreement with those retrieved from the single-atom, three-step, quantum-mechanical model depicted in Fig. 3(c). In these calculations the spectral phase and amplitude distribution of the short and long trajectories have been retrieved by the three-step, single-atom-response, quantum-mechanical model. For $I_{\ell}$ in the plateau region, the harmonics were taken with equal beam diameters [see Fig. 1(d)], while when the harmonics enter in the cutoff region the harmonic beam profile does not influence the interference structure since the two trajectories degenerate into one with a single phase. The "saw-type" structure disappears when the 


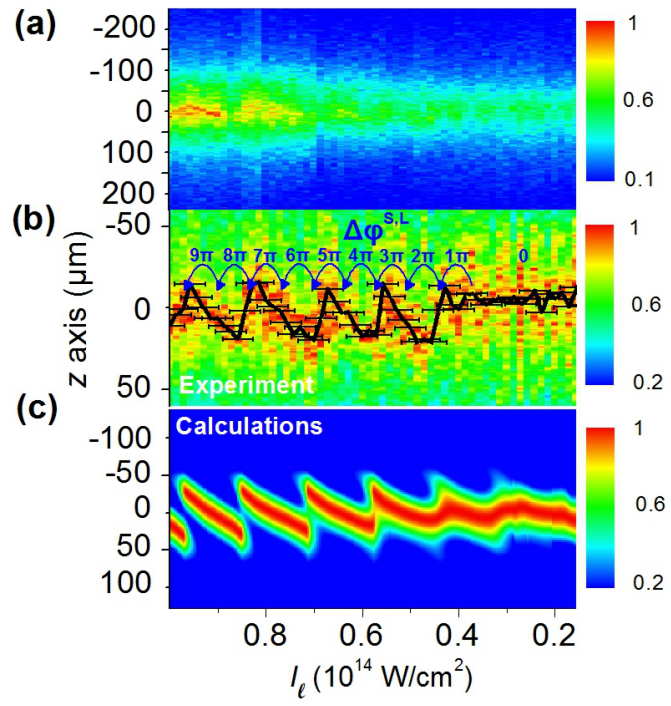

FIG. 3. (Color online) Imaging the intensity-dependent spatial EUV interference pattern. (a) Contour plot showing the dependence of the interference pattern along the $z$ axis on $I_{\ell}$. The relative amplitudes of the 11th, 13th, and 15th harmonics in the interaction region are shown in Fig. 1(b). (b) Contour plot retrieved after normalization of the plot in (a) to equal ion-signal amplitudes. The black line depicts the mean value of the ion distribution. The error bars represent the standard deviation of the mean value resulting by taking into account the accuracy of the laser intensity measurement. (c) Calculated (and normalized) contour plot showing the dependence of the $z$-axis interference pattern on $I_{\ell}$. Although the "saw-type" structure and the position of the maxima on the $z$ axis are in fair agreement with the measured values, this calculation is based on rough assumptions (e.g., single-atom response, flat spatial harmonic phase distributions) and is only meant to provide a qualitative description of the data.

EUV emission consists of only the short or the long trajectory harmonics.

The measured "saw-type" structure can provide direct access to the mechanism underlying the harmonic generation process [Fig. 4(a)], since the difference between harmonic emission times $\left(\Delta t_{e}=t_{e}^{L_{q}}-t_{e}^{S_{q}}\right)$ and the corresponding difference $\Delta L_{e}=L_{e}^{L_{q}}-L_{e}^{S_{q}}$ between "long" and "short" path lengths can be directly measured. An advantage of the present approach as compared to interferometric approaches [25] is that any dispersion effect, which may be introduced by the ionization in the harmonic generation region, is physically absent in the measurement. This is because all of the information about the harmonic emission time differences is obtained by measuring the phase differences between the "short" and the "long" trajectories contributing to the same harmonic generated by the same EUV source and not between different harmonics generated by two independent EUV sources. It should be mentioned that transient phase-matching effects [23], resulting in spectral modifications, are not expected to alter the findings of the present work, because the scheme used in our measurements spectrally integrates the detected radiation. Here, the values of $\Delta t_{e}$ have been deduced from the "saw-type" structure by using the following considerations: (a) $\Delta \varphi_{q}^{S, L}=0$ at lower values of $I_{\ell}$, where an $I_{\ell}$ independent single-peak structure is observed. This is in agreement with the harmonic generation theory, where in the cutoff region the two trajectories degenerate into one with a single phase. (b) $\Delta \varphi_{q}^{S, L}$ is increasing monotonically with $I_{\ell}$ [38]. (c) The $\Delta \varphi_{q}^{S, L}$ is increasing by $\pi$ when the structure changes from a single to a double peak. Following these considerations, the values of $\Delta \varphi_{q}^{S, L}$ with differences $n \pi(n=0,1,2, \ldots)$ and $\Delta t_{e}=\Delta t_{e}^{q}=$ $n T_{q} / 2$ as a function of $I_{\ell}$ have been obtained and are shown in Figs. 4(b) [shown also schematically in Fig. 3(b)] and 4(c). $T_{q} / 2$ is the half period of the $q$ th harmonic. By using the approximate relation $\phi_{q}^{S, L}\left(I_{\ell}\right) \approx-U_{p} \tau_{q}^{S, L}=-a_{q}^{S, L} I_{\ell}$ (where $\alpha_{q}^{S, L}$ is the phase coefficient of the $q$ harmonic emitted by short and long trajectories) for the phases of the emitted harmonics and the measurements of Fig. 4(b), it can be shown that, in case of xenon at the high $I_{\ell}$ range, $\Delta \phi_{q}^{S, L}\left(I_{\ell}\right) \approx$ $-\Delta a_{q}^{S, L} I_{\ell} \approx 44 \times 10^{-14}\left(\operatorname{rad~cm}{ }^{2} / \mathrm{W}\right) \delta I_{\ell}\left(\mathrm{W} / \mathrm{cm}^{2}\right)$ (for $q=$ 11-15 and $\left.\Delta \alpha_{q}^{S, L}=\alpha_{q}^{S}-\alpha_{q}^{L}\right)$. Although the value $\Delta \alpha_{q}^{S, L}$ for argon, krypton, and neon has been demonstrated before [22,23,25,39-41], to our knowledge, for xenon, there is a lack of information. The value of $\Delta \alpha_{q}^{S, L}$ obtained in the present study is in fair agreement with the theoretical predictions for the dominance of the two "shortest" ("short" and "long") electron trajectories [19,39-41] and the recent experimental findings of Ref. [23], while is larger by a factor of $\approx 3$ compared to previously reported experimental values for argon and krypton [25]. At $I_{\ell}$ regions, where more than one harmonic lies in the plateau, $\Delta t_{e}$ reflects the average value of the emission time differences weighted by the harmonic amplitude $\left(E_{q}\right)$, i.e., $\Delta t_{e}=\left\langle\Delta t_{e}^{q}\right\rangle=\sum_{q} E_{q} \Delta t_{e}^{q} / \sum_{q} E_{q}$. In terms of electron path length, the difference between the trajectories can be obtained by the relation $\Delta L_{e}=\sqrt{2\left(E_{e}+V_{i}\right) / m_{e}} \Delta t_{e}$ (where $E_{e}+V_{i}=\hbar \omega_{q}$ is the final kinetic energy of the recolliding electron, $E_{e}$ is the return energy of the electron, and $V_{i}$ is the binding energy of the atom) $[16,18]$. For a single recollision $0 \leqslant E_{e} \leqslant 3 U_{p}$ [18], the above relation can be approximated by $\Delta L_{e} \approx \sqrt{2\left(1.5 U_{p}+V_{i}\right) / m_{e}} \Delta t_{e}\left(U_{p}\right.$ is the ponderomotive energy of the electron). An interesting observation from the measured data is the correlation of the emission time differences with the electron quantum path interferences. Extracting the ratio $f\left(I_{\ell}\right)=\Delta L_{e} / \lambda_{e}$ of $\Delta L_{e}$ to the electron de Broglie wavelength $\left[\lambda_{e}=h / \sqrt{2 m_{e}\left(1.5 U_{p}+V_{i}\right)}\right]$ [Fig. 4(d)], it is found that for emission time differences $\Delta t_{e} \approx n T_{q} / 2$, the electron quantum path difference is changing by $\Delta L_{e} \approx$ $n \lambda_{e}$. This is clearly shown in the intensity dependence of the differences $\Delta f\left(I_{\ell}\right)=f\left(I_{\ell}^{(i)}\right)-f\left(I_{\ell}^{(i+1)}\right), i=1 \ldots 12$, between consecutive values of $f\left(I_{\ell}\right)$, at which $\Delta f\left(I_{\ell}\right) \approx 0$ and $\Delta f\left(I_{\ell}\right) \approx \lambda_{e}$ for the cutoff and the plateau, respectively [Fig. 4(e)]. The small drift of $\Delta f\left(I_{\ell}\right)$ from the mean value 1 is mainly due to a deviation of $E_{e}$ from the value of $1.5 U_{p}$. From these measurements, it is concluded that in the intensity range around $I_{\ell} \approx 7 \times 10^{13} \mathrm{~W} / \mathrm{cm}^{2}$, a phase change of $2 \pi$ between the "short" and "long" electron wave packets is taking place for $\mathrm{a} \approx 12 \%$ intensity variation and leads to a $\pi$ phase difference between the radiation emitted by the "short" and "long" trajectories. Moreover, significant information about the harmonic generation process arises from the observation that when $I_{\ell}$ is increased by $\approx 1.5 \times 10^{13} \mathrm{~W} / \mathrm{cm}^{2}$, an energy of $2 \hbar \omega_{\ell}$ is absorbed by the system and an additional higher harmonic enters into the plateau regime. This 2-IR-photon energy increases the electron momentum by $2 \hbar k_{\ell}\left(k_{\ell}\right.$ is the 


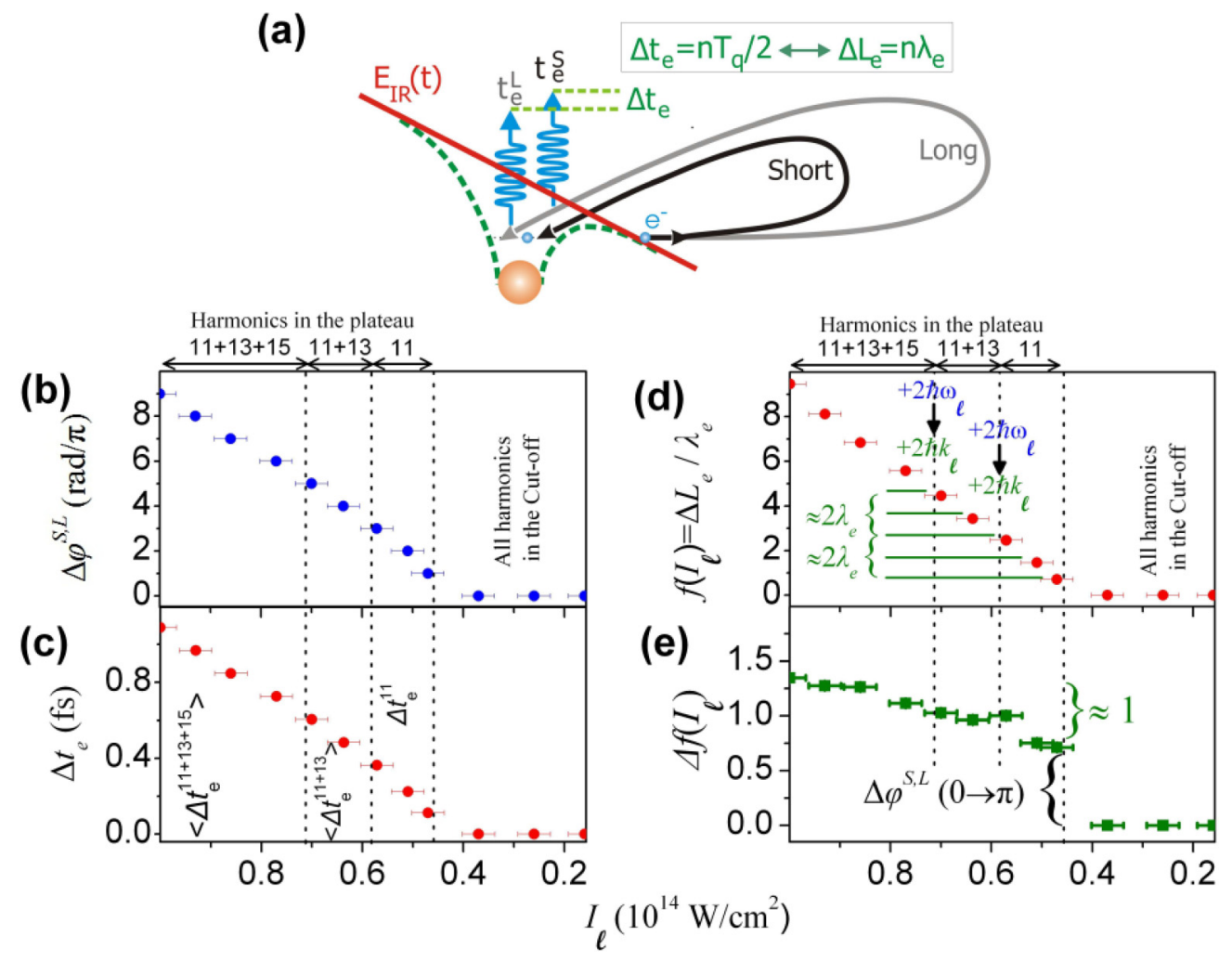

FIG. 4. (Color online) Measurement of harmonic emission time and electron path-length differences. (a) High-order-harmonic generation mechanism shown in the spirit of the "three-step model" $[18,20,21]$. The IR field suppresses the atomic potential and allows the valence electron to tunnel through. The electron moves almost freely in the driving field, gaining kinetic energy, which is converted to photons upon its recombination. Due to the different electron trajectories, the harmonic emission times are different. $t_{e}^{S}$ and $t_{e}^{L}$ correspond to the harmonic emission times resulting from the "short" and "long" trajectories, respectively. (b) Measured phase difference between the "short" and "long" trajectories as a function of $I_{\ell}$. (c) Difference between the emission times $\Delta t_{e}=t_{e}^{S}-t_{e}^{L}$. (d) Path-length difference $\Delta L_{e}$ normalized by the de Broglie electron wave $\lambda_{e}$. For emission time differences $\Delta t_{e} \approx n T_{q} / 2$, the electron quantum path differences are changing by $\Delta L_{e} \approx n \lambda_{e}$. (e) Difference between the consecutive values of $f\left(I_{\ell}\right)$. In all graphs, the vertical black dotted lines depict the harmonic cutoff regions at which the 2-IR-photon absorption increases the electron momentum by $2 \hbar k_{\ell}$ and the error bars represent one standard deviation of the mean value.

wave number of the IR photon) and results in a trajectory path-length difference increase of $2 \lambda_{e}$ [Fig. 4(d)].

Here we would like to point out that the values shown in Fig. 4 constitute a direct outcome of the measurement, meaning that $\Delta \varphi_{q}^{S, L}$ is the overall phase difference of the short and long trajectory harmonics, which, according to the three-step model, is introduced in the recollision process by the ionization, recombination, and the electron motion in the continuum. Once the harmonics have been emitted, this phase difference can be simply translated into a time difference of emission times, i.e., $\Delta \varphi_{q}^{S, L}=\omega_{q} \Delta t_{e}$. In the spirit of the three-step model, the measured phase difference $\Delta \varphi_{q}^{S, L} \approx \omega_{q} \Delta t_{r}^{S, L}-\Delta S^{S, L}\left(p, t_{r}^{S, L}, \tau^{S, L}\right)$ (where $p$ is the electron momentum drift, $S$ is the action along the path, $\tau^{S, L}=t_{r}^{S, L}-t_{i}^{S, L}$ is the traveling time of the electron in the continuum, and $t_{i}^{S, L}, t_{r}^{S, L}$ are the ionization and recombination times of the $q$ th "short" and "long" trajectory harmonics). Thus, the measured $\Delta t_{e}$ is the overall emission time difference of the short and long trajectory harmonics (delay in the photoemission between the short and long trajectories due to $\Delta \varphi_{q}^{S, L}$ ) introduced in the recollision process, i.e., $\Delta t_{e} \approx$ $\Delta t_{r}^{S, L}-\Delta S^{S, L}\left(p, t_{r}^{S, L}, \tau^{S, L}\right) / \omega_{q}$.
Extending our technique to nonlinear probing schemes using a target gas with a higher ionization potential (helium) could further enhance the precision of the measurements via the enhancement of the fringe visibility in the EUVinterference pattern. The main advantage of the two-photon scheme compared to the single-photon one is a substantial reduction of the background ion signal which limits the visibility of the interference pattern in the single-photon ionization image. Initial experiments in which helium is used to image the EUV focus via a two-photon nonresonant ionization process demonstrate a big step towards this goal. It has been found that the overall width and the contrast of the ion distribution pattern are consistent with the order of the nonlinearity of the process. Nevertheless, further investigation is required in this direction since the noise level of the measurement does not permit an unambiguous statement on the observed interference pattern.

\section{CONCLUSIONS}

In the present work, by using a high spatial resolution ion imaging detector, we have been able to produce a clear picture of focused EUV radiation generated in a gas-phase medium. In 
this way a close look into the harmonic generation process has been able to deduce the spatial EUV-field distribution from the spatial ion distribution produced in the EUV focal area. Interference patterns of the spatial intensity distribution of the EUV field, resulting from "long" and "short" trajectory contributions, are mapped by the spatially resolved ion yield. By manipulating the intensity-dependent electron paths we have measured the difference between the harmonic emission times and the electron path lengths resulting from different electron trajectories. In this way, a quantitative relation between the emission times and electron path lengths (for $\Delta t_{e}=n T_{q} / 2$ the change of $\Delta L_{e}$ is $n \lambda_{e}$ ) has been obtained while the IR energy transfer to the electron momentum has also been observed. Thus, it has been found that a phase change of $2 \pi$ between the "short" and "long" electron wave packets occurs for $\mathrm{a} \approx 12 \%$ intensity variation and leads to a $\pi$ phase difference between the radiation emitted by the "short" and "long" trajectories. Based on these findings, we have revealed the quantum nature of the recollision process. Also, the approach can shed light on several strong-field light-matter interaction processes resulting in coherent light emission, including harmonic generation from atoms and molecules, surface plasma, and bulk crystals [7-9,42-46]. Attosecond science [1-5,10-12], high-resolution spectroscopy studies in the EUV [13-15] spectral region, and molecular tomography methods [7-9] are among the research topics that can benefit from the in situ control of the emitted EUV phase distribution and/or the spatial selection of the EUV-radiation-atom interaction products.

\section{ACKNOWLEDGMENT}

This work is supported in part by the European Commission programs ATTOFEL, CRISP, Laserlab Europe, the Greek funding program NSRF, and performed in the framework of the DAAD-IKYDA program for the promotion of scientific cooperation between Greece and Germany.
[1] P. Agostini and F. L. DiMauro, Rep. Prog. Phys. 67, 813 (2004).

[2] P. B. Corkum and F. Krausz, Nat. Phys. 3, 381 (2007).

[3] F. Krausz and M. Ivanov, Rev. Mod. Phys. 81, 163 (2009).

[4] J. M. Dahlström, A. L'Huillier, and A. Maquet, J. Phys. B 45, 183001 (2012).

[5] P. Tzallas, E. Skantzakis, J. E. Kruse, and D. Charalambidis, in Progress in Ultrafast Intense Laser Science, Springer Series in Chemical Physics Vol. VII (Springer, Berlin, 2011), p. 163.

[6] D. Shafir, Y. Mairesse, D. M. Villeneuve, P. B. Corkum, and N. Dudovich, Nat. Phys. 5, 412 (2009).

[7] J. Itatani, J. Levesque, D. Zeidler, H. Niikura, H. Pépin, J. C. Kieffer, P. B. Corkum, and D. M. Villeneuve, Nature (London) 432, 867 (2004).

[8] S. Haessler, J. Caillat, W. Boutu, C. Giovanetti-Teixeira, T. Ruchon, T. Auguste, Z. Diveki, P. Breger, A. Maquet, B. Carré, R. Taieb, and P. Salieres, Nat. Phys. 6, 200 (2010).

[9] C. Vozzi, M. Negro, F. Calegari, G. Sansone, M. Nisoli, S. De Silvestri, and S. Stagira, Nat. Phys. 7, 822 (2011).

[10] G. Sansone, F. Kelkensberg, J. F. Pérez-Torres, F. Morales, M. F. Kling, W. Siu, O. Ghafur, P. Johnsson, M. Swoboda, E. Benedetti, F. Ferrari, F. Lépine, J. L. Sanz-Vicario, S. Zherebtsov, I. Znakovskaya, A. L'Huillier, M. Yu. Ivanov, M. Nisoli, F. Martín, and M. J. J. Vrakking, Nature (London) 465, 763 (2010)

[11] P. Tzallas, E. Skantzakis, L. A. A. Nikolopoulos, G. D. Tsakiris, and D. Charalambidis, Nat. Phys. 7, 781 (2011).

[12] E. Goulielmakis, Z.-H. Loh, A. Wirth, R. Santra, N. Rohringer, V. S. Yakovlev, S. Zherebtsov, T. Pfeifer, A. M. Azzeer, M. F. Kling, S. R. Leone, and F. Krausz, Nature (London) 466, 739 (2010).

[13] C. Gohle, T. Udem, M. Herrmann, J. Rauschenberger, R. Holzwarth, H. A. Schüssler, F. Krausz, and T. W. Hänsch, Nature (London) 436, 234 (2005).

[14] D. Z. Kandula, C. Gohle, T. J. Pinkert, W. Ubachs, and K. S. E. Eikema, Phys. Rev. Lett. 105, 063001 (2010).

[15] A. Cingöz, D. C. Yost, T. K. Allison, A. Ruehl, M. E. Fermann, I. Hartl, and J. Ye, Nature (London) 482, 68 (2012).
[16] D. Shafir, H. Soifer, B. D. Brunner, M. Dagan, Y. Mairesse, S. Patchkovskii, M. Yu. Ivanov, O. Smirnova, and N. Dudovich, Nature (London) 485, 343 (2012).

[17] O. Raz, O. Pedatzur, B. D. Bruner, and N. Dudovich, Nat. Photonics 6, 170 (2012).

[18] M. Lewenstein, P. Balcou, M. Y. Ivanov, A. L'Huillier, and P. B. Corkum, Phys. Rev. A 49, 2117 (1994).

[19] P. Salieres, B. Carré, L. Le Déroff, F. Grasbon, G. G. Paulus, H. Walther, R. Kopold, W. Becker, D. B. Milosevic, A. Sanpera, and M. Lewenstein, Science 292, 902 (2001).

[20] P. B. Corkum, Phys. Rev. Lett. 71, 1994 (1993).

[21] K. J. Schafer, B. Yang, L. F. DiMauro, and K. C. Kulander, Phys. Rev. Lett. 70, 1599 (1993).

[22] A. Zaïr, M. Holler, A. Guandalini, F. Schapper, J. Biegert, L. Gallmann, U. Keller, A. S. Wyatt, A. Monmayrant, I. A. Walmsley, E. Cormier, T. Auguste, J. P. Caumes, and P. Salières, Phys. Rev. Lett. 100, 143902 (2008).

[23] C. M. Heyl, J. Güdde, U. Höfer, and A. L'Huillier, Phys. Rev. Lett. 107, 033903 (2011).

[24] J. E. Kruse, P. Tzallas, E. Skantzakis, and D. Charalambidis, Phys. Rev. A 82, 033438 (2010).

[25] C. Corsi, A. Pirri, E. Sali, A. Tortora, and M. Bellini, Phys. Rev. Lett. 97, 023901 (2006).

[26] E. Constant, D. Garzella, P. Breger, E. Mével, C. Dorrer, C. Le Blanc, F. Salin, and P. Agostini, Phys. Rev. Lett. 82, 1668 (1999).

[27] E. Takahashi, Y. Nabekawa, T. Otsuka, M. Obara, and K. Midorikawa, Phys. Rev. A 66, 021802(R) (2002).

[28] T. Ditmire, J. K. Crane, H. Nguyen, L. B. DaSilva, and M. D. Perry, Phys. Rev. A 51, R902(R) (1995).

[29] P. Balcou, P. Salières, A. L'Huillier, and M. Lewenstein, Phys. Rev. A 55, 3204 (1997).

[30] M. B. Gaarde and K. J. Schafer, Phys. Rev. Lett. 89, 213901 (2002).

[31] M. Bellini, C. Lyngå, A. Tozzi, M. B. Gaarde, T. W. Hänsch, A. L'Huillier, and C.-G. Wahlström, Phys. Rev. Lett. 81, 297 (1998). 
[32] P. Salieres, T. Ditmire, M. D. Perry, A. L'Huillier, and M. Lewenstein, J. Phys. B 29, 4771 (1996).

[33] P. Salières, A. L. Huillier, and M. Lewenstein, Phys. Rev. Lett. 74, 3776 (1995).

[34] E. J. Takahashi, H. Hasegawa, Y. Nabekawa, and K. Midorikawa, Opt. Lett. 29, 507 (2004).

[35] M. Schultze, B. Bergues, H. Schröder, F. Krausz, and K. L. Kompa, New J. Phys. 13, 033001 (2011).

[36] Kaesdorf S Geräte für Forschung und Industrie, Gabelsbergerstr. 59, D-80333 München, Germany.

[37] M. Born and E. Wolf, Principles of Optics (Pergamon, New York, 1968).

[38] Y. Mairesse, A. de Bohan, L. J. Frasinski, H. Merdji, L. C. Dinu, P. Monchicourt, P. Breger, M. Kovacev, R. Taieb, B. Carré, H. G. Muller, P. Agostini, and P. Salieres, Science 302, 1540 (2003).

[39] M. B. Gaarde, J. L. Tate, and K. J. Schafer, J. Phys. B 41, 132001 (2008).
[40] M. Lewenstein, P. Salieres, and A. L'Huillier, Phys. Rev. A 52, 4747 (1995).

[41] K. Varjú, Y. Mairesse, B. Carré, M. B. Gaarde, P. Johnsson, S. Kazamias, R. López-Martens, J. Mauritsson, K. J. Schafer, Ph. Balcou, A. L'Huillier, and P. Salières, J. Mod. Opt. 52, 379 (2005).

[42] U. Teubner and P. Gibbon, Rev. Mod. Phys. 81, 445 (2009).

[43] J. Seres, V. S. Yakovlev, E. Seres, Ch. Streli, P. Wobrauschek, Ch. Spielmann, and F. Krausz, Nat. Phys. 3, 878 (2007).

[44] S. Ghimire, A. D. DiChiara, E. Sistrunk, P. Agostini, L. F. DiMauro, and D. A. Reis, Nat. Phys. 7, 138 (2010).

[45] A. Paul, R. A. Bartels, R. Tobey, H. Green, S. Weiman, I. P. Christov, M. M. Murnane, H. C. Kapteyn, and S. Backus, Nature (London) 421, 51 (2003).

[46] F. Quéré, C. Thaury, P. Monot, S. Dobosz, P. Martin, J.-P. Geindre, and P. Audebert, Phys. Rev. Lett. 96, 125004 (2006). 